\title{
BMJ Open Organisational best practices for advancing women in leadership: protocol for a systematic literature review
}

\author{
Mariam Mousa (D) , ${ }^{1,2}$ Alexandra K Mullins, ${ }^{1}$ Helen Skouteris, ${ }^{1,3}$ Jacqueline Boyle, ${ }^{1,4}$ \\ Helena J Teede ${ }^{1,2}$
}

To cite: Mousa M,

Mullins AK, Skouteris $\mathrm{H}$, et al. Organisational best practices for advancing women in leadership: protocol for a systematic literature review. BMJ Open 2021;11:e046982. doi:10.1136/ bmjopen-2020-046982

- Prepublication history and additional supplemental material for this paper are available online. To view these files, please visit the journal online (http://dx.doi.org/10.1136/ bmjopen-2020-046982)

Received 15 November 2020 Revised 10 March 2021 Accepted 09 April 2021

Check for updates

(c) Author(s) (or their employer(s)) 2021. Re-use permitted under CC BY-NC. No commercial re-use. See rights and permissions. Published by BMJ.

${ }^{1}$ Monash Centre for Health Research and Implementation, School of Public Health, Monash University, Melbourne, Victoria, Australia

${ }^{2}$ Monash Partners Academic Health Science Centre, Member of the Australian Health Research Alliance, Melbourne, Victoria, Australia

${ }^{3}$ University of Warwick, Coventry, UK

${ }^{4}$ Epworth HealthCare,

Melbourne, Victoria, Australia

Correspondence to

Dr Helena J Teede;

Helena.Teede@monash.edu

\section{ABSTRACT}

Introduction Evidence-based approaches for achieving gender equity for women in leadership are lacking. Current efforts are of limited effectiveness, especially in healthcare. This work occurs in the context of an Australian National Health and Medical Research Council funded partnership to advance women in healthcare leadership. Partners include government, professional colleges and healthcare organisations with national reach and international links. Here we present a protocol for a systematic review, aiming to capture evidence on effective organisational strategies across multiple sectors with comparable challenges in advancing women in leadership. The aim of the review is to learn from other sectors and analyse the evidence to inform implementation in the health sector.

Methods and analysis A systematic search will be performed on Ovid MEDLINE, PsycINFO and SCOPUS databases to identify studies since 2000 , reflecting a major shift in the global gender equality agenda with the development of the 2000 Millennium Development Goals. Titles and abstracts will be screened to assess eligibility; data extraction, quality assessment (using the Critical Appraisal Skill Programme checklist) and synthesis of outcomes will be performed. Outcomes will be assessed using the Grading of Recommendations, Assessment, Development and Evaluation framework. Studies in English using quantitative or qualitative design, which investigate organisational practices in any sector, for advancing women in leadership, and report on one or more measurable outcomes (eg, capacity-building, incidence of promotion) will be included. Findings will be analysed, themes will be extracted and results will be described.

Ethics and dissemination Ethics approval is not required. To our knowledge, this review will be the first to provide a comprehensive synthesis of available evidence on organisational practices for advancing women in leadership from the last two decades. Findings will be published in peer-reviewed journals and disseminated at conferences and meetings. Through a large-scale funded partnership, this work will inform practice, linking to international initiatives.

PROSPERO registration number CRD42020162115; International Prospective Register of Systematic Reviews.
Strengths and limitations of this study

- This systematic review aims to capture the breadth of research on organisational approaches, strategies and practices from multiple industry sectors, which advance women in leadership. Evidence is sought from published, peer-reviwed literature (2000-2020).

- This study underpins a large-scale government, healthcare and professional society partnership programme to capture, analyse, translate and implement effective strategies on advancing women in healthcare leadership.

- The protocol is registered and risk of bias is assessed using the Critical Appraisal Skills Programme checklist.

- Limitations may include heterogeneity of study methods and outcomes, limiting feasibility of meta-analysis.

- Dissemination and impact will be significant, informing national and international healthcare organisational strategies through an established funded partnership.

\section{INTRODUCTION}

The underrepresentation of women in top positions of leadership is a persistent phenomenon in the health sector. Genderbased barriers that prevent women from advancing to leadership positions are known and thoroughly understood. ${ }^{1-4}$ Yet, despite this research from industries, including business, government, education, academia, medicine and healthcare, similar patterns of under-representation of women in leadership persist as do gender-based attitudes and barriers. ${ }^{245}$ In fact, women are often underleveraged as a source of leadership in nearly all industries and across all occupations from board members and executives to Chief Executive Officer (CEO) positions and College presidents. ${ }^{1}{ }^{6}$ In industries dominated by women, such as healthcare, 
the rules of engagement have been established decades ago, aligned with traditional male leadership paradigms. ${ }^{7}$ Here, individualistic leadership styles, associated with traditionally masculine representations of leadership, dominate the discourse and are enacted in a hierarchical manner, rendering any change to the status quo a highly challenging endeavour. ${ }^{68}$ Efforts targeting women, such as career planning, while well-intentioned, have largely focused on individual capability alone, while missing the systemic and organisational changes necessary to ensure that both men and women have equal opportunity to lead organisations to success. ${ }^{7}$ As a result, and despite the support for change, women in healthcare continue to earn less, have less opportunities and less promotions than their male counterparts. ${ }^{7-12}$ A focus on the gendered status of women requires closer attention, including differences across professional silos. This will provide insight into the workings of gender in healthcare leadership, with implications.

Recent research has yielded key insights, ${ }^{13}$ where only a small minority of respondents reported that women are not interested in, or suited to leadership. Indeed, women bring qualities to the workplace that are well suited for transforming healthcare to have better outcomes. ${ }^{14-17}$ For instance, research shows that women are empathetic collaborators who value team work and bring expertise to building relationships that encourage others to reach their potential. ${ }^{13-15} 1819$ These qualities enhance other skills commonly associated with traditionally masculine leadership styles, including risk-taking, and assertiveness, ${ }^{1162021}$ and bring to the table a diverse perspective, new insights, ideas and skills that lead to better problem solving and improved performance within an organisation. ${ }^{162} 22$ To support this, research also shows that businesses with a gender-diverse leadership team and higher representation of women on boards had, on average, higher financial performance than those with lower representations of women. ${ }^{7}$ Yet, despite this research across industries, including businesses, government, education, academia, medicine and healthcare, ${ }^{2} 5132324$ the same patterns of under-representation of women in leadership positions persist along with the same gender-based attitudes and barriers that perpetuate the status quo.

Gender-related barriers to advancing women into leadership reflect wide-ranging themes of limited workforce capacity, limited opportunities to improve capability and persistent gendered attitudes towards women's credibility as leaders. ${ }^{2} 41323-26$ A focus on the gendered status of women also requires closer attention to intersecting statuses that can reveal important heterogeneity within the group and provide insight into the workings of gender in the production of healthcare patterns, with implications for policy and practice response. ${ }^{27}$ Indeed, in the healthcare sector, women play a significant role in the delivery of health services, yet they remain minority decision-makers, holding less than $25 \%$ of the most influential positions, with few opportunities for advancement. $^{245}$ Much of the female healthcare workforce is either underpaid or unpaid, and they continue to experience higher rates of sexism, gender-based discrimination and bias with inequitable attitudes towards their credibility and capability to lead. ${ }^{245}$

Today, we are amidst an optimistic disruption that is challenging dominant discourses around gender and the systemic inequities that perpetuate them. Herein lies an opportunity to optimise organisational effort and improve outcomes for women by providing the necessary evidence synthesis to establish what works, and to inform future action for implementing and scaling gender equity-specific practices that promote the advancement of women in leadership. ${ }^{28}{ }^{29}$ This systematic review protocol outlines the aims to capture and synthesise evidence across sectors, on effective organisational strategies for advancing women in leadership. Here, we include studies of any design, targeting any age, ethnicity or geographic location, which describe organisational level interventions, addressing gender equity in leadership.

\section{SYSTEMATIC REVIEW QUESTION}

What are the organisational level strategies and practices that advance women in leadership across sectors to inform organisational reform in healthcare?

\section{METHODS/DESIGN}

This review will conform to the standards of the Preferred Reporting Items for Systematic Reviews ${ }^{30}$ as outlined in the online supplemental material 1.

\section{Eligibility criteria}

The eligibility of studies will be determined via the selection criteria established using the Population, Intervention, Comparison, Outcomes framework outlined in table 1 .

\section{Search strategy}

A systematic search using relevant terms is outlined in the online supplemental material 2. The following electronic databases will be searched. The database selection was pragmatic to cover the broader fields where interventions on advancing women in leadership are focused.

- MEDLINE via OVID/Medline in-process and other nonindexed citations via OVID.

- PsycINFO via OVID.

- SCOPUS.

\section{Study selection}

Studies published between 2000 and 2020 will be selected for review. This timeframe reflects a major shift in the global agenda for gender equality, where the leadership gap was actively addressed with the release of the UN's Millennium Development Goals.

To further determine eligible studies, the titles, abstracts and keywords of retrieved studies will be reviewed by two reviewers against the selection criteria outlined in table 2. 
Table 1 PICO for study inclusion

\begin{tabular}{|c|c|c|c|c|}
\hline & Participants (P) & Intervention (I) & Comparison (C) & Outcomes (0) \\
\hline Study types & & \multicolumn{3}{|c|}{ Quantitative and qualitative study designs } \\
\hline Language & & \multicolumn{3}{|l|}{ English } \\
\hline
\end{tabular}

PICO, Population, Intervention, Comparison, Outcomes.

Studies that meet the inclusion criteria will be retrieved for full-text review.

\section{Data extraction}

The reviewer (MM) will extract data from all included studies. Pilot testing of the extraction form will be conducted (by MM and AKM) using 3-4 studies to ensure all relevant data are captured. Where available, data as specified in table 2 will be extracted in aggregate format from all studies. For each study group, extracted data will include sample sizes, aggregate point estimates, measures of variability and frequency counts for dichotomous variables. Continuous variables will be collected as aggregate mean values with SD. Outcomes reported as dichotomous variables will be extracted as relative measures of risk (risk ratio or OR along with CIs) as well as proportions or absolute numbers of participants experiencing the outcome of interest.

\section{Quality appraisal of the evidence}

Risk of bias will be assessed by two reviewers at the study level. Based on the study design, the appropriate template from the Critical Appraisal Skills Programme will be used to assess risk of bias. Each study will be allocated a high, moderate or low-risk of bias rating. Individual quality items will be assessed using a descriptive component approach that includes items such as the presence of prespecified selection criteria, sample sizes, appropriate controls or comparison groups, participant blinding where applicable. Methods of outcome assessment and reporting, including statistical issues such as powering and data analysis, will also be included. Disagreement will be resolved through mediation with senior authors.

\section{Data synthesis and analysis}

For all studies, data will be synthesised from all sectors for comparison and presented in summary tables and in narrative form to describe the populations, interventions and outcomes of the included studies. Where quantitative data are available, between-group or within-group differences will be presented, and relative differences in outcomes will be assessed. Aggregate effect measures will be used for meta-analyses if appropriate, when data are derived from homogenous groups (where participants, interventions and outcome measures are sufficiently similar), using random-effects models in Review Manager V.5.3. Statistical heterogeneity will be assessed using the $\mathrm{I}^{2}$ test, where $\mathrm{I}^{2}$ values over $50 \%$ indicate moderate to high heterogeneity. Descriptive analyses will be conducted

Table 2 Aggregate data to be extracted from included studies

\begin{tabular}{llll}
\hline Study & Participants & Intervention & Outcomes \\
\hline $\begin{array}{l}\text { First author and journal/ } \\
\text { source }\end{array}$ & $\begin{array}{l}\text { Women of any age and ethnicity } \\
\text { if available }\end{array}$ & $\begin{array}{l}\text { Workplace practices, } \\
\text { approaches, policies or } \\
\text { procedures }\end{array}$ & $\begin{array}{l}\text { Capacity-building, perceptions of } \\
\text { credibility capability and confidence* }\end{array}$ \\
$\begin{array}{l}\text { Country and year of } \\
\text { publication }\end{array}$ & $\begin{array}{l}\text { Other SES* or relevant } \\
\text { characteristics of participants }\end{array}$ & $\begin{array}{l}\text { Practical guidelines, models } \\
\text { and tools }\end{array}$ & $\begin{array}{l}\text { Incidence of promotion, retention and } \\
\text { representation of women* }\end{array}$ \\
$\begin{array}{l}\text { Study design, setting, } \\
\text { duration and sample size } \\
\text { and sample description }\end{array}$ & $\begin{array}{l}\text { Career stage, early/ mid/late } \\
\text { and position descriptions }\end{array}$ & $\begin{array}{l}\text { Organisational frameworks, } \\
\text { strategies }\end{array}$ & $\begin{array}{l}\text { Other measurable outcomes relevant } \\
\text { to career mobility/progression into } \\
\text { leadership }\end{array}$ \\
\hline
\end{tabular}

*As defined by authors of the studies.

SES, Socioeconomic status . 
for those studies that are deemed too heterogeneous or present insufficient data (eg, outcomes measured in single studies) for pooling or combined analysis. Subgroup analyses will be performed for factors presumed to cause variations in outcomes and may include age, ethnicity, seniority or career stage, sector or setting or other factors deemed relevant during the review process.

Qualitative data will be analysed using thematic analysis, as a method of identifying patterns across large bodies of data. This method of analysis is characterised by theoretical flexibility and is thus, often incorporated into multiple research methods (e.g. narrative or discursive), which makes it useful for working with data across several theoretical frameworks. ${ }^{31}$ The process involves searching across patterns in the data, which will then be clustered for the description of emergent themes ${ }^{31}$ within the broader context of women in leadership. In that sense, we intend to take a critical framework approach to the analysis with the aim of exposing and decolonising the discourse that underlies how organisations approach gender equity for women in leadership.

\section{Grading the body of evidence at the outcome level}

Where possible, the quality of the evidence will be assessed by two reviewers using the Grading of Recommendations, Assessment, Development and Evaluation approach. ${ }^{32}$ Based on multiple components (eg, risk of bias, heterogeneity), ratings will be assigned to each outcome as high, moderate, low or very low quality. Where needed, consensus will be sought through consultation with a third independent reviewer.

\section{Public involvement statement}

In the context of the Australian National Health and Medical Research Council (NHMRC) partnership programme, stakeholders will be engaged to inform priorities and assist in the translation, dissemination and implementation of the outcomes from this study.

\section{Ethics and dissemination}

This systematic review will provide the most comprehensive update of available evidence on organisational approaches, practices and policies for advancing women in leadership from multiple industry sectors. Consistent with the vision of the leading organisation, the Monash Centre for Health Research and Implementation, this work follows an implementation science framework. ${ }^{33}$ The systematic review is foundational in a larger funded national programme that involves broad stakeholder partnership and engagement. Results of this review will be published in peer-reviewed journals and disseminated at conferences. A key dissemination aim here is that this work will be a valuable resource that informs government and organisational policy decisions and implementation. Internationally, results will be disseminated through the Women in Global Health International Network https:/ / www.womeningh.org/. This work will underpin further significant research. Ethics approval is not required for this secondary research.

\section{DISCUSSION}

A recent report by the World Economic forum ${ }^{79-12}$ states that the failure to fully realise gender equity and promote women's potential in the labour market is a key contributor to the slowing down of economic growth globally. ${ }^{79-12}$ Gender equity in this instance refers to the application of 'fairness of treatment for all, according to their respective needs', which includes equal or different, yet equivalent treatment across rights, benefits, obligations and opportunities. ${ }^{23}$ Equity is critical for workforce diversity, encouraging differences to create a productive environment where everyone is valued and their skills are optimised and harnessed. ${ }^{23}$ In turn, a diverse workforce represents society, understands and responds to community, ${ }^{23}$ embodies the principles of equity and fairness and models the diffusion of prejudices, thus promoting a discrimination-free environment and transforming workplace culture. Never has this been more important to embrace than in the healthcare sector today. While there has been some progress towards gender equity in the sector, it remains slow and in need of evidence-based efforts that inform healthcare organisations on gender equity strategies. ${ }^{28}{ }^{29}$ The growing number of women in the health sector is not reflected in the representation of women in healthcare leadership, ${ }^{4}$ regardless of role as Dean, Chief Medical Officer, College board or committee member or CEO of a large hospital. ${ }^{13}$ This continued restriction of the goals of women, and inability to harness their education and potential as leaders, as well as a lack of diversity in agenda-setting for matters related to healthcare outcomes, can inevitably result in major implications affecting the health of populations. ${ }^{2} 1328$

The broad case for advancing women in leadership and enhancing gender equity is compelling. The specific needs in the healthcare sector are clear and yet there is a lack of clarity on evidence-based approaches to advancing women in leadership broadly across sectors and within healthcare. To progress change, there is a need to move towards actively championing equity at an organisational and policy/systems level. Effective, evidence-based and measurable organisational approaches and practices that advance women in leadership are now vital. This review will synthesise knowledge and identify organisational strategies and key gaps for further research. Importantly it will directly inform and underpin a large-scale national implementation research, evaluation and scale-up programme with international links to drive gender equity in healthcare leadership.

\section{Strengths and limitations}

The proposed systematic literature review provides an opportunity to address the issue of gender inequity in leadership by bringing together a team of multidisciplinary experts. In this partnership, the team will inform 
the next steps following evidence synthesis, by establishing what works and how to implement best practice for gender equity and improve outcomes for women in healthcare leadership. ${ }^{28}{ }^{29}$ To do this, we aim to integrate this team of experts, with government partners, multiple health professional colleges and health services for whom this initiative is prioritised, to codesign, develop and implement solutions for the healthcare sector. It is anticipated that this systematic review will deliver a comprehensive synthesis of organisational approaches, practices and policies for advancing women in leadership. The proposed body of work will follow rigorous methodology and will involve acquiring and synthesising data from studies published over the past two decades in multiple industry sectors. Some limitations of the systematic review process include the potential for publication bias since we only include peer-reviewed, published and English language studies. The quality of studies in the field may be moderate and variably heterogeneous, thereby affecting the strengths of the conclusions drawn from the review. Nevertheless, the work will bring together cross-industry learnings and establish a foundation for future research in this field. Future research will be enabled through a large-scale Australian NHMRC-funded partnership project, together with government policy-makers, Professional Medical, Nursing and Allied Health Colleges, large public and private health services and multidisciplinary academics with leading experts in health, business and leadership, evidence synthesis, implementation, measurement and evaluation.

\section{Twitter Mariam Mousa @MariamMousa}

Contributors MM co-designed and drafted this review protocol and will conduct the search and screening and coordinate the systematic review process. HJT co-designed the protocol, contributed to writing and editing the manuscript, and will supervise the review process. HJT is the study guarantor and will oversee data collection, synthesis and analysis. All authors, including AKM, HS and JB, provided content expertise and support for the protocol methods and search strategy and reviewed and edited the manuscript. All authors have contributed substantial intellectual input in line with ICMJE criteria for authorship and have approved the final version of the protocol for publication.

Funding This research and the overarching and broader programme of work is funded by an NHMRC partnership grant [ID 1198561]. MM and AKM are supported by an Epworth Healthcare, Cabrini Healthcare and Monash University academic scholarships. JB is supported by an NHMRC Career Development Research Fellowship and HJT by an NHMRC Medical Research Future Fund Practitioner Fellowship.

Competing interests None declared.

Patient consent for publication Not required.

Provenance and peer review Not commissioned; externally peer reviewed.

Supplemental material This content has been supplied by the author(s). It has not been vetted by BMJ Publishing Group Limited (BMJ) and may not have been peer-reviewed. Any opinions or recommendations discussed are solely those of the author(s) and are not endorsed by BMJ. BMJ disclaims all liability and responsibility arising from any reliance placed on the content. Where the content includes any translated material, BMJ does not warrant the accuracy and reliability of the translations (including but not limited to local regulations, clinical guidelines, terminology, drug names and drug dosages), and is not responsible for any error and/or omissions arising from translation and adaptation or otherwise.

Open access This is an open access article distributed in accordance with the Creative Commons Attribution Non Commercial (CC BY-NC 4.0) license, which permits others to distribute, remix, adapt, build upon this work non-commercially, and license their derivative works on different terms, provided the original work is properly cited, appropriate credit is given, any changes made indicated, and the use is non-commercial. See: http://creativecommons.org/licenses/by-nc/4.0/.

ORCID iD

Mariam Mousa http://orcid.org/0000-0001-7547-9261

\section{REFERENCES}

1 McDonagh KJ, Paris NM. The leadership labyrinth: Leveraging the talents of women to transform health care. Nurs $A d m Q$ 2013;37:6-12.

2 Clark J, Zuccala E, Horton R. Women in science, medicine, and global health: call for papers. Lancet 2017;390:2423-4.

3 Timmins N. Leading for integrated care: 'If you think competition is hard, you should try collaboration'. The King Fund, 2019.

4 ACHE. A comparison of the career attainments of men and women healthcare executives. Chicago, IL: Division of Member Services, American College of Healthcare Executives, 2012.

5 Ibarra HE. Women rising: the unseen barriers. Harv Bus Rev 2013.

6 Currie G, Spyridonidis D. Sharing leadership for diffusion of innovation in professionalized settings. Human Relat 2019;72:1209-33.

7 Elias E. Lessons learned from women in leadership positions. Work 2018;59:175-81.

8 Timmins N. Leading for integrated care: 'If you think competition is hard, you should try collaboration'. The Kind Fund, 2019.

9 Kuhlmann E, Ovseiko PV, Kurmeyer C, et al. Closing the gender leadership gap: a multi-centre cross-country comparison of women in management and leadership in academic health centres in the European Union. Hum Resour Health 2017;15:2.

10 Kulich C, Ryan MK, Haslam SA. Where is the romance for women leaders? the effects of gender on leadership attributions and performance-based pay. Appl Psychol 2007;56:582-601.

11 O'Neil DA, Hopkins MM. The impact of gendered organizational systems on women's career advancement. Front Psychol 2015;6:905.

12 Shen $\mathrm{H}$. Inequality quantified: mind the gender gap. Nature 2013;495:22-4.

13 Bismark M, Morris J, Thomas L, et al. Reasons and remedies for under-representation of women in medical leadership roles: a qualitative study from Australia. BMJ Open 2015;5:e009384.

14 Hauser MC. Leveraging women's leadership talent in healthcare. $J$ Healthc Manag 2014;59:318-22.

15 Lowe M. Book reviews : breaking the glass ceiling: can women reach the top of America's largest corporations ? , 1988: 19, 145-7.

16 Matoff-Stepp S, Applebaum B, Pooler J, et al. Women as health care decision-makers: implications for health care coverage in the United States. J Health Care Poor Underserved 2014;25:1507-13.

17 Forum WE. The global gender gap report. Geneva, 2017.

18 Cermak JH, Jeeves J. Women in leadership: lessons from Australian companies leading the way Australia. The Business Council of Australia, McKinsey \& Company \& Workplace Gender Equality Agency, 2017.

19 Koenig AM, Eagly AH, Mitchell AA, et al. Are leader stereotypes masculine? A meta-analysis of three research paradigms. Psychol Bull 2011;137:616-42.

20 McDonagh KJ, Bobrowski P, Hoss MAK, et al. The leadership gap: ensuring effective healthcare leadership requires inclusion of women at the top. OJL 2014;03:20-9.

21 WEF. The global gender gap report. Geneva, 2017.

22 Lowe M. Breaking the glass cieling: Can women reach the top of America's largest corporations? In: Management education and development. , 1988: 19, 145-7.

23 Du travail B, Date-Bah E, Zhang J, et al. ABC of women workers' rights and gender equality. Geneva: International Labour Office, 2000.

24 Santen RJ, Joham A, Fishbein L, et al. Career advancement: meeting the challenges confronting the next generation of endocrinologists and endocrine researchers. J Clin Endocrinol Metab 2016;101:4512-20.

25 AlHW. Medical workforce 2011: National health workforce series no.3. Canberra: Australian Institute of Health and Welfare, 2011.

26 Weiss A, Lee KC, Tapia V, et al. Equity in surgical leadership for women: more work to do. Am J Surg 2014;208:494-8.

27 Bates LM, Hankivsky O, Springer KW. Gender and health inequities: a comment on the final report of the who Commission on the social determinants of health. Soc Sci Med 2009;69:1002-4. 
28 Carnes M, Bairey Merz CN. Women are less likely than men to be ful professors in cardiology: why does this happen and how can we fix it? Circulation 2017;135:518-20.

29 Du travail B, Date-Bah E, Zhang J. ABC of women workers' rights and gender equality. 2 edn. Geneva: International Labour Office, 2007.

30 Stewart LA, Clarke M, Rovers M, et al. Preferred reporting items for systematic review and meta-analyses of individual participant data: the PRISMA-IPD statement. JAMA 2015;313:1657-65.
31 Clarke V, Braun V. Thematic analysis. J Posit Psychol 2017;12:297-8.

32 Atkins D, Best D, Briss PA, et al. Grading quality of evidence and strength of recommendations. BMJ 2004;328:1490.

33 Mills J, Yates $\mathrm{K}$, Harrison $\mathrm{H}$, et al. Using a community of inquiry framework to teach a nursing and midwifery research subject: an evaluative study. Nurse Educ Today 2016;43:34-9. 